\title{
Le financement du secteur de l'huile de palme : pourquoi les institutions financières de développement doivent continuer à investir en Afrique*
}

\author{
Thomas SKURTIS ${ }^{1}$ \\ Guédi AÏNACHÉ ${ }^{1}$ \\ Didier SIMON² \\ 1 Proparco (Division Entreprises), \\ groupe Agence française de développement, \\ 151, rue Saint-Honoré, \\ 75001 Paris, France \\ <skurtist@proparco.fr> \\ $<$ ainacheg@proparco.fr> \\ 2 Division agriculture et développement rural \\ de I'Agence française de développement, \\ 5, rue Roland Barthes, \\ 75598 Paris Cedex 12, \\ France \\ <simond@afd.fr>
}

\begin{abstract}
Over the past three decades, palm oil has become the leading vegetable oil, a commodity that can be found in a large variety of food and non-food products. Mainly produced in South-East Asia, palm oil has recently been at the centre of many controversies. The industry has sometimes grown in an unsustainable way, with negative environmental and social impacts, among which deforestation, loss of biodiversity, poor working conditions in industrial plantations or land grabbing. In September 2009, the recognition of these issues has led the International Finance Corporation (IFC, member of the World Bank Group) to stop all its investments in the sector worldwide. This example has been followed by most development finance institutions (DFls) which have put on hold any investment in South-East Asia and are now very cautious in the rest of the world. However, the bad Asian experience should not prevent DFls from investing in this sector in Africa. The continent lacks from local production despite the importance of this oil in its diet. But palm oil is not only a question of food security. When properly supervised, the industry can also have a significant impact on rural development. It creates local added value and jobs. Palm oil producers often provide social services (heath, education etc.) that Governments do not always offer in remote rural areas. Moreover, through the rehabilitation of old and non performing plantations, the industry has room for an environment friendly growth. It is the role of DFls to support sponsors of palm oil projects while insisting on their commitment to best practices and the most stringent environmental and social criteria.
\end{abstract}

Key words: Palm oil, Africa, food security, rural development, environmental and social responsability fait aujourd'hui l'huile végétale la plus produite dans le monde. Elle vient essentiellement de deux pays, I'Indonésie, devenue premier producteur mondial en $2007^{1}$, et la Malaisie, qui fournissent plus de $85 \%$ de la production mondiale, soit 38,5 millions de tonnes (figure 1). Cette très forte croissance de production s'explique principalement par la compétitivité de l'huile de palme vis-à-vis des autres huiles végétales grâce à ses rendements agricoles bien plus importants. En moyenne, le palmier à huile permet de produire 3,8 tonnes d'huile par hectare, soit neuf fois plus que le soja, huit fois plus que le colza et six fois plus que le tournesol ${ }^{2}$. Cependant, le secteur de l'huile de palme est aujourd'hui très controversé. Alors que ses supporters affirment qu'il s'agit d'une industrie durable, seule capable d'alimenter un monde consommant toujours plus, ses détracteurs l'accusent d'être la source d'une multitude de problèmes, notamment environnementaux et sociaux.

En premier lieu, les ONG de conservation de la nature dénoncent la poursuite à grande échelle de la destruction d'écosystèmes tropicaux

\footnotetext{
*Les opinions et propos exprimés dans cet article n'engagent que leurs auteurs et leur publication ne signifie pas que Proparco ou I'AFD souscrivent aux opinions qui y sont exprimées.
}

humides (forêts, tourbières) d'Asie du Sud-Est d'importance majeure pour la biodiversité mondiale, notamment en Indonésie. La disparition progressive de l'orang-outan est ainsi devenue le symbole des effets du développement du palmier à huile. II est indéniable que l'extension des plantations d'huile de palme a causé une déforestation et une fragmentation des forêts très préjudiciables à la pérennité de ces écosystèmes.

L'huile de palme fait aussi polémique dans le secteur social. Les conditions de travail des ouvriers agricoles dans certaines grandes plantations industrielles sont mauvaises (contrats de travail précaires, travail des enfants, absence de couverture médicale, conditions de sécurité parfois insuffisantes, rémunération à la tâche inéquitable). Sur le plan foncier, la gouvernance locale ne permet pas toujours d'assurer l'exercice de droits traditionnels, entraînant de nombreux conflits entre les petits exploitants, les communautés locales, les peuples autochtones et les grands producteurs à la recherche de terres pour l'établissement de plantations industrielles. Enfin, comme de nombreuses matières premières agricoles, I'huile de palme peut être transformée en biocarburant. Sa forte productivité en fait d'ailleurs, avec la canne à sucre, I'une des 


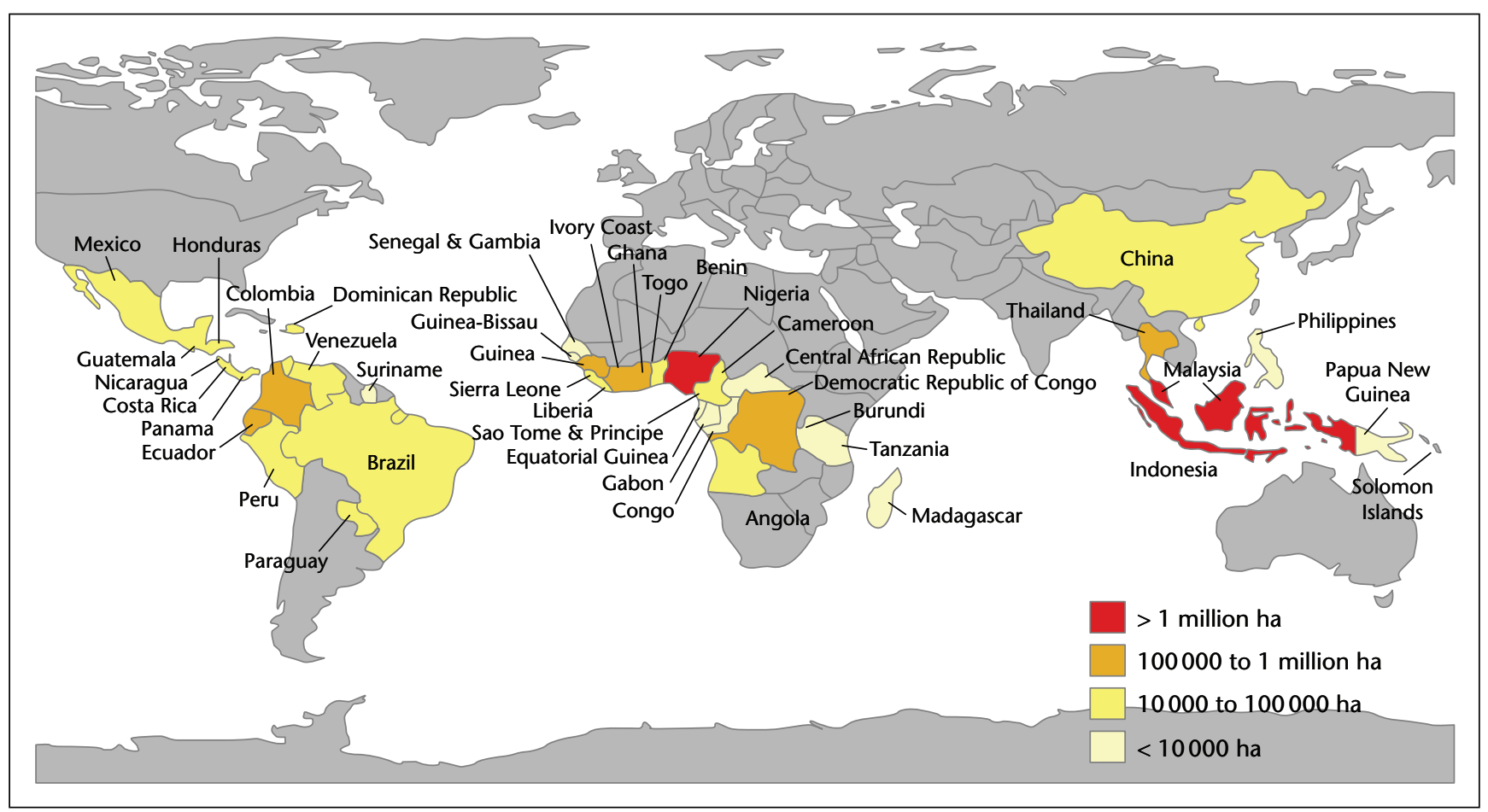

Figure 1. Les plantations de palmier à huile au niveau mondial (source : FAO).

cultures les plus rentables au plan énergétique. La mise en place de cadres règlementaires et une conjoncture de prix favorables pourraient induire le développement du palmier à huile pour la production de biodiesel, au détriment des productions alimentaires et des milieux naturels.

\section{Pourquoi, malgré tout, financer le secteur de I'huile de palme en Afrique?}

\section{Importance de l'huile de palme en Afrique}

Ces controverses ont conduit de nombreuses institutions de développement à revoir leur position sur le financement de ce secteur. Après avoir injecté près d'un milliard de dollars depuis 1965, la Société financière internationale (SFI) a décidé de surseoir à toutes ses opérations en déclarant, en septembre 2009, un moratoire mondial sur le financement de I'huile de palme. Un processus de consultation de l'ensemble des acteurs du secteur (bailleurs, $\mathrm{ONG}$, industriels, etc.) visant à redéfinir son cadre d'intervention sectoriel a été lancé ${ }^{3}$. Ses conclusions sont attendues prochainement. La communauté des institutions de

\footnotetext{
${ }^{3}$ Voir www.ifc.org/palmoilstrategy
}

développement a emboité le pas à la SFI en Asie du Sud-Est. Sans y avoir totalement stoppé ses interventions, elle a de plus adopté une attitude très prudente dans le reste du monde, notamment en Afrique.

Cependant, si ces controverses concernent avant tout I'Asie du Sud-Est, principal centre de production et d'exportation n'ayant pas su maîtriser la croissance du secteur, la réalité africaine est bien différente. Freiner les investissements sur ce continent ne ferait qu'entraver un développement nécessaire qui peut se faire dans des conditions satisfaisantes (figure 2).

Alors que l'Afrique était leader de l'offre mondiale d'huile de palme dans l'entredeux-guerres, elle ne représente aujourd'hui que $5,6 \%$ de sa production, partagée entre le Nigeria $(54,7 \%)$ et une douzaine d'autres pays. Son poids dans les exportations est encore plus faible : environ $1 \%{ }^{4}$.

La production africaine d'oléagineux est très inférieure à la consommation locale. Ainsi, dans l'ensemble des 16 pays de la Cedeao ${ }^{5}$, le déficit est estimé actuellementà 500000 tonnes. Comblé par les importations provenant des pays asiatiques, ce déficit atteindra 1,5 million

\footnotetext{
${ }^{4}$ Sources : Banque mondiale, FAOSTAT

${ }^{5}$ Communauté économique des États de l'Afrique de l'Ouest: Bénin, Burkina Cap-Vert, Côte-d'Ivoire, Gambie, Ghana, Guinée, Guinée-Bissau, Liberia, Mali, Niger, Nigeria, Sénégal, Sierra Leone, Togo.
}

de tonnes en $2020^{6}$. Or, les oléagineux, essentiellement les huiles végétales, entrent pour $15 \%$ dans la ration alimentaire des populations africaines qui atteint en moyenne, selon l'analyse d'Agrimonde, $1800 \mathrm{kcal}$ par personne et par jour?.

Dans cette perspective, l'huile de palme mérite une attention particulière, d'autant plus que, au-delà de sa prédominance sur le marché mondial des huiles végétales, elle est en moyenne $30 \%$ moins chère que les huiles concurrentes (tableau 1).

\section{Une gestion durable}

du secteur de l'huile de palme peut avoir de très forts impacts sur le développement de l'Afrique

Compte tenu de son importance dans le régime alimentaire de certains pays d'Afrique, du déficit de production locale et de son faible coût, le développement des filières oléagineux et notamment de I'huile de palme est un impératif de sécurité alimentaire.

\footnotetext{
${ }^{6}$ Source : R. Hirsch, 2003.

${ }^{7}$ La consommation annuelle moyenne $d$ 'huile de palme par habitant est de $23,8 \mathrm{~kg}$ dans le monde, $59,3 \mathrm{~kg}$ aux États-Unis, $51,7 \mathrm{~kg}$ dans I'UE et $12,5 \mathrm{~kg}$ au Nigeria.
} 


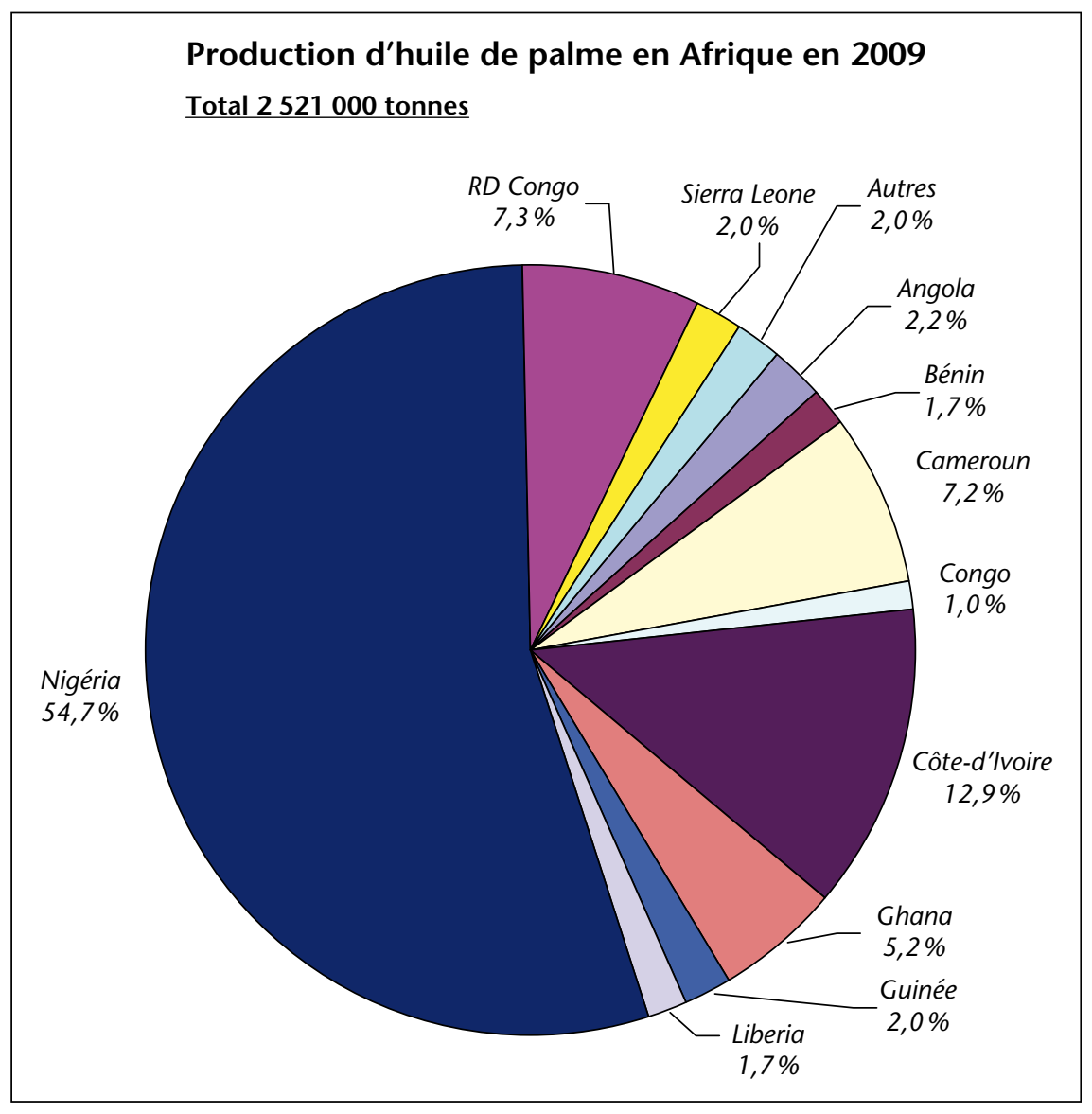

Figure 2. Production d'huile de palme en Afrique en 2009.

Tableau 1. Moyenne annuelle du prix des principales huiles en dollars/tonne.

\begin{tabular}{|llllllll|}
\hline Graisses et huiles & $\mathbf{2 0 0 3}$ & $\mathbf{2 0 0 4}$ & $\mathbf{2 0 0 5}$ & $\mathbf{2 0 0 6}$ & $\mathbf{2 0 0 7}$ & $\mathbf{2 0 0 8}$ & $\mathbf{2 0 0 9}$ \\
\hline Huile de palme & 443 & 471 & 422 & 478 & 780 & 948 & 682 \\
\hline Huile de soja & 553 & 616 & 544 & 598 & 881 & 1258 & 848 \\
\hline Huile d'arachide & 1243 & 1161 & 1060 & 970 & 1347 & 2105 & 1138 \\
\hline Huile de tournesol & 593 & 684 & 677 & 658 & 1021 & 1498 & 854 \\
\hline
\end{tabular}

Source : FAOSTAT.

C'est, en outre, une opportunité de développement si des stratégies durables sont mises en œuvre.

Au niveau macroéconomique, la substitution d'importations par une production locale permet de créer de la valeur ajoutée et de réduire le déséquilibre de la balance commerciale.

Au niveau local, I'huile de palme contribue également à la création de revenus et d'emplois au sein du monde rural où réside une partie importante des populations pauvres, permettant ainsi de limiter l'exode rural en offrant des perspectives de long terme. Le cas spécifique des petits planteurs villageois est à ce titre très assurent aux planteurs des revenus plus élevés que le revenu journalier agricole moyen et permettent aux industriels de sécuriser un approvisionnement régulier pour leurs usines. Enfin, ces relations contractuelles peuvent contribuer à l'équipement des zones rurales concernées. Les producteurs industriels prennent souvent à leur charge la construction ou la rénovation d'infrastructures (pistes, électrification) mais aussi la fourniture de services sociaux (dispensaires, écoles, campagnes de prévention contre le sida) que l'État n'assure pas toujours.

L'impact environnemental du développement de la production d'huile de palme semble pouvoir être mieux maîtrisé en Afrique. En effet, ce développement peut se faire par la réhabilitation et la modernisation de très nombreuses plantations existantes ainsi que par la mise en valeur de terres arables non couvertes par les forêts primaires. Le remplacement, par des clones plus productifs, de plantations établies depuis plusieurs dizaines d'années et dont les rendements sont décroissants permet d'améliorer la production sans déforestation ni perte de biodiversité. Par ailleurs, dans la plupart des plantations africaines, industrielles comme villageoises, les performances techniques et économiques sont aujourd'hui inférieures à celles des pays asiatiques. Cela est notamment dû à l'âge des plantations et aux mauvaises pratiques d'entretien et d'exploitation des palmiers. La marge de progrès est donc très importante et la mise en place de programmes agronomiques adaptés permet $d^{\prime}$ envisager une augmentation de production pouvant atteindre 10 à $20 \%$ sans impact sur l'environnement.

Bien entendu, dès lors que des institutions de développement soutiennent le développement de cette filière en Afrique, elles doivent s'assurer de la mise en œuvre de plans d'actions conformes aux meilleures pratiques et standards internationaux. En 2004, une partie de I'industrie de I'huile de palme s'est organisée en association (la $\mathrm{RSPO}^{8}$ ) pour établir des principes et critères de production durable qui interdisent notamment de nouvelles plantations sur les terres abritant des forêts à haute valeur de conservation. En 2008, la SFI a adopté des standards encore plus contraignants 9 .

elles peuvent être liées par des contrats, assurent dans certains pays une part importante de la production ( $80 \%$ au Nigeria et $65 \%$ en Côte-d'Ivoire). La contractualisation entre les plantations villageoises et les industriels présente de multiples avantages : accès au crédit, accès à des intrants de qualité, conseils techniques et prévisibilité de revenus. D'une façon générale, des contrats équilibrés entre planteurs villageois et partenaires industriels

\footnotetext{
${ }^{8}$ Voir Roundtable on Sustainable Palm Oil. www. rspo.org

${ }^{9}$ Voir à ce titre les critères de performance environnementale et sociale de la SFI : http://www.ifc.org/ ifcext/sustainability.nsf/AttachmentsByTitle/pol SocEnvSustainability2006_French/\$FILE/Sustainability Policy_French.pdf
} 


\section{Encadré 1}

\section{Proparco}

Proparco est une institution financière de développement, conjointement détenue par l'Agence française de développement (AFD) et par des actionnaires privés du Nord et du Sud. La société a pour mission de catalyser les investissements privés dans les pays émergents et en développement en faveur de la croissance, du développement durable et de l'atteinte des objectifs du millénaire (OMD). Née il y a 30 ans de la conviction que le secteur privé est un acteur clé du développement, Proparco a triplé son capital en 2008. Proparco finance des opérations économiquement viables, socialement équitables, soutenables sur le plan environnemental et financièrement rentables. Sa stratégie sectorielle, adaptée au niveau de développement des pays, se concentre sur le secteur productif, les systèmes financiers, les infrastructures et le capital investissement. La société investit dans un champ géographique allant des grands pays émergents aux pays les plus pauvres, notamment en Afrique, avec un degré élevé d'exigence en matière de responsabilité sociale et environnementale. Proparco propose une palette complète d'instruments financiers permettant de répondre aux besoins spécifiques des investisseurs privés dans les pays en développement (prêts, fonds propres, garanties et ingénierie financière). En 2009, Proparco a octroyé 1,1 milliard d'euros à travers 80 projets dans plus de trente pays.

Pour le groupe AFD, les points critiques d'éligibilité d'un projet concernent principalement : - la responsabilité sociale des producteurs visà-vis de leurs employés, y compris ceux embau-

\section{Encadré 2}

\section{L'Agence française de développement}

Établissement public, l'Agence française de développement (AFD) agit depuis près de 70 ans pour combattre la pauvreté et favoriser le développement dans les pays du Sud et dans l'outre-mer. Elle met en œuvre la politique définie par le Gouvernement français.

Présente sur le terrain dans plus de 50 pays et dans neuf collectivités d'outre-mer, l'AFD finance et accompagne des projets qui améliorent les conditions de vie des populations, soutiennent la croissance économique et protègent la planète: scolarisation, santé maternelle, appui aux agriculteurs et aux petites entreprises, adduction d'eau, préservation de la forêt tropicale, lutte contre le réchauffement climatique, etc.

En 2009, I'AFD a consacré plus de 6,2 milliards d'euros au financement d'actions dans les pays en développement et émergents et en faveur de l'outre-mer. Ils ont notamment contribué à la vaccination de 1,8 million d'enfants, I'amélioration de l'approvisionnement en eau potable pour 7,3 millions de personnes et le soutien à 900000 emplois dans le secteur productif. Les projets d'efficacité énergétique sur la même année permettront d'économiser près de cinq millions de tonnes de $\mathrm{CO}_{2}$ par an.

chés par des sous-traitants. Une attention particulière est notamment apportée à la rémunération, la qualité des logements, la scolarisation des enfants résidant sur les plantations, la santé du personnel, le régime de retraite, etc. ;

- la responsabilité des producteurs industriels vis-à-vis des agriculteurs liés par contrats d'approvisionnement (prix d'achat des régimes, prix des prestations d'appui technique, prix des intrants, etc.) ;

- les conditions d'acquisition ou de location des terres et, dès lors que celles-ci relèvent d'une gestion communautaire, la nature des compensations accordées à ces communautés (loyers, infrastructures, etc.) ;

- le plan de gestion environnemental des plantations industrielles comme villageoises en termes de préservation ou restauration des corridors de biodiversité, de contrôle des contaminations des cours d'eau par les résidus de produits phytosanitaires ou $d$ 'engrais et les effluents des usines, d'économie d'énergie, etc.

\section{Conclusion}

Les débats sur des conditions de productions de l'huile de palme par certaines compagnies dans certains pays asiatiques ne doivent pas conduire à différer le développement de cette filière en Afrique. Des modèles de croissance durables et responsables existent qui peuvent contribuer à l'indépendance et à la sécurité alimentaire de l'Afrique, à la création d'emplois et à la diversification des revenus en zones rurales. Les institutions de développement, en soutenant la croissance du secteur, doivent veiller à ce que les meilleures options environnementales et sociales soient mises en œuvre. Il conviendra toutefois de veiller à ce que la priorité reste donnée à la sécurité alimentaire et à ce que le développement du biodiesel à partir de I'huile de palme ne remette pas en cause cette priorité. 